\title{
G scment \\ Adherence to DASH Dietary Pattern and Polycystic Ovarian Syndrome: A Case-control Study
}

asieh panjeshahin

Shahid Sadoughi University of Medical Sciences and Health Services: Shahid Sadoughi University of Medical Sciences and Health Services https://orcid.org/0000-0003-0434-5463

\section{Akram Ghadiri-Anari}

Shahid Sadoughi University of Medical Sciences and Health Services: Shahid Sadoughi University of Medical Sciences and Health Services

Mahdieh Hosseinzadeh ( $\sim$ hoseinzade.mahdie@gmail.com )

Shahid Sadoughi University of Medical Sciences and Health Services: Shahid Sadoughi University of Medical Sciences and Health Services https://orcid.org/0000-0001-7482-2494

\section{Research note}

Keywords: DASH diet, polycystic ovary syndrome, Diet, Body composition, BMI

Posted Date: June 25th, 2021

DOl: https://doi.org/10.21203/rs.3.rs-645997/v1

License: (c) (i) This work is licensed under a Creative Commons Attribution 4.0 International License. Read Full License 


\section{Abstract}

Objective: Polycystic Ovary Syndrome (PCOS) as one of the endocrine and metabolic abnormalities is prevalent in reproductive-aged women. This study aimed to investigate the relevance between adherence to the DASH diet and PCOS.

Results: This is an age and BMI-matched case-control study including $(\mathrm{n}=108)$ new diagnosis PCOS women as a case group and women without PCOS as a control group $(n=108)$. Also, the validated 168item food frequency questionnaire was used to determine the usual dietary intake. The method of Fung et al. was used to calculate the DASH score. Moreover, logistic regression was assessed to evaluate the relationship between adherence to the DASH diet and the odds of PCOS. After adjustment for potential confounders, an increase in adherence to the DASH diet significantly reduced the odds of PCOS (OR for the highest vs. lowest quartile $=0.18 ; 95 \% \mathrm{Cl} 0.07,0.47)$. There was a significant decreasing trend in the odds of PCOS across increasing quintiles of the DASH diet ( $\mathrm{p}$-trend $<0.001)$. The results showed that adherence to the DASH diet could reduce the odds of PCOS by 82 percent. It's worth noting that more prospective studies are required to confirm the findings of the current study.

\section{Introduction}

Polycystic ovary syndrome (PCOS) is one of the most multifactorial disorders and frequent endocrine abnormalities among women in reproductive age [1, 2], which prevalence between 10-15\%, depending on diagnostic criteria [3, 4]. Based on the Rotterdam criteria, the prevalence of PCOS in Iran has been reported to be 19.5\% [5]. Infertility, endometrial carcinoma, anxiety, impaired hemorrhage, sleep apnea, and metabolic disorders such as obesity, hypertension, type 2 diabetes, impaired gonadotropin secretory activity, insulin resistance, cardiovascular diseases, and metabolic syndrome may be complications of this syndrome [6-10]. Previous studies have indicated that adherence to physical activity and diet treatment recommendations are very important to achieve therapeutic goals [11]. A combination of using medication and lifestyle modifications are treatment plans for this chronic condition [12]. Given that the exact etiology of PCOS is not clear yet, but the interactions between genetic and environmental factors such as diet are considered as effective factors on PCOS [13-16]. Therefore, diet management and weight loss as lifestyle modifications, have been suggested as the primary strategy in the treatment of PCOS $[17,18]$.

Dietary Approaches to Stop Hypertension (DASH) dietary pattern is a low-glycemic index and low energydense diet which has firstly been considered for the regulation of blood pressure [19]. This dietary pattern is rich in vegetables, fruits, whole grains, and low-fat dairy with a reduced content of sodium, saturated and total fat[19]. Therefore, due to these useful ingredients, favorable effects of the DASH diet on insulin resistance, lipid profiles, biomarkers of inflammation and oxidative stress, and PCOS women have also been reported repeatedly [20-23]. Besides, Some studies indicated beneficial effects of the DASH diet on androgens, antioxidant status, body composition, weight loss, AMH (anti-Müllerian hormone), lipid, and metabolic profiles in PCOS women $[21,24,25]$. Also, a randomized controlled trial study conducted by 
Azadi-Yazdi et al. indicated that adherence to the DASH diet had beneficial effects on androstenedione, SHBG, and antioxidant capacity in women with PCOS [26]. DASH diet contains higher amounts of dietary fiber, phytoestrogens, potassium, calcium, magnesium, folic acid, and other beneficial nutrients [20, 27], which justifies the beneficial effects of this dietary pattern on PCOS. For example, the useful effect of high-fiber and phytoestrogens food on the regulation of testosterone levels in women with PCOS have been mentioned before [28]. As well as, this diet is rich in monounsaturated fatty acids which improve glycemic control and lipid profiles by improves insulin sensitivity [29]. Another related mechanism is the beneficial effect of magnesium on incremental of SHBG level, which correlates with insulin sensitivity and decreases the free fraction of androgens [30]. Therefore, investigating the DASH diet based on other dietary quality indices to clarify other useful features of this healthy diet, will be helpful.

To the best of our knowledge, this study is the first investigation aimed to evaluate the association between adherence to the DASH diet and odds of PCOS in new diagnosed patients in Middle Eastern countries. Also, we adjusted for a wide range of confounding variables including body composition such as body fat percentage in addition to energy intake.

\section{Materials And Methods}

\section{Study design and participants}

This age-BMI matched case-control study was done on women with and without PCOS, to evaluate the association between adherence to the DASH diet and odds of polycystic ovarian syndrome. The participants were selected among those who referred to "Yazd Diabetes Clinic" Iran, from January 2018 to March 2019. Moreover, the endocrinology expert introduced several participants from different clinics in all parts of the city, who had the inclusion criteria. Also, the Rotterdam criteria was used for diagnosing women with PCOS [3]. The participants' recruitment procedures are indicated in Figure S1.

Two groups were age and BMI matched using a frequency matching method. After explained the study process, a signed written informed consent was provided by each participant.

\section{Dietary intake assessment}

Each participant using a 178-items semi-quantitative food frequency questionnaire (FFQ) with 551 questions by common dietary intake of the recent year was assessed. This FFQ was an adjusted model of the previously validated 168-item food frequency questionnaire, which was validated in the Tehran Lipid and Glucose Study (TLGS). Ten additional questions related to the consumption of Yazd specific food items were added to the original 168-item FFQ leading to a questionnaire with 178 items.

A face to face interview with individuals was conducted by a blinded dietitian to fill out all of the questionnaires. To increase the precision and accuracy of the estimations, the portion sizes, and the frequency of food intake and beverage items, on average, were questioned. Then, the frequency was transformed into daily consumption, and portion sizes were converted to gram via household 
measurements. Finally, the actual food intake (g/day) was transferred to Nutritionist IV software (First Databank Inc., Hearst Corp., San Bruno, CA, USA) which is modified for Iranian foods to compute the total energy and nutrients intake. The overall energy and nutrients intake were investigated by transferring food intake (g/day) to Nutritionist IV.

\section{Calculation of adherence to DASH diet indices}

The DASH score was calculated to check the participant's adherence to the DASH diet using the method of Fung et al. [31] based on foods and nutrients that emphasized and focusing on eight components in the DASH diet: low intake of sugar-sweetened beverages and sweets, red and processed meats, and sodium, and a high intake of fruits, vegetables, whole grain, nuts and legumes, and low-fat dairy products, according to quintile categories. A score of 5 was given to participants with the highest quintile of low-fat dairy products, vegetables, fruits, nuts and legumes, and whole grains and a score of 1 was given to participants with the lowest quintile of mentioned foods. Also, Participants with a lower intake of sweetened beverages, sodium, and red and processed meats got higher points (ie, the score of 5 was for the highest quintile and the score of 1 was for the lowest quintile). Eventually, the score of adherence to the DASH diet was calculated for each participant by Sum of the scores, ranging from 8 to 40 .

\section{Statistical analysis}

Participants were divided into quartiles according to their scores of adherence to the DASH diet.

Comparing quantitative variables between cases and controls conducted by using an independent t-test whereas, for categorical variables, chi-square ( $\chi 2$ test) was used. Kolmogorov-Smirnov test conducted to examine whether the distribution of variables is normal or not. Mean \pm SD were presented for continuous variables while qualitative variables used frequency (percentage).

We compared the characteristics of participants (by Classification in quartiles) in the lowest and highest quartiles of adherence to the DASH diet scores using descriptive statistics. One-way ANOVA was used to compare general characteristics of quantitative variables across quartiles of adherence to the DASH diet, and for categorical variables used $\chi 2$ tests. We calculated Odds Ratio (OR) and $95 \%$ confidence interval (Cl) for crude and adjusted models.

We used multivariable logistic regression in different models to evaluate the odds ratio for each of the three upper quartiles of adherence to the DASH diet (compared with the lowest quartile) and the prevalence of PCOS. In all of the analyses, the first quartile of adherence to the DASH diet scores was considered as a reference. To assess the overall trend of odds ratios across increasing quartiles of adherence to DADH diet, we treated the quartile categories as an ordinal variable in the analyses. All analyses were conducted using the statistical software IBM SPSS Statistics version 24. P values $<0.05$ considered statistically significant.

\section{Results}




\section{Characteristics of the study population}

Characteristics of the subjects across quartiles of adherence to the DASH diet scores are indicated in Table 1. As you can see in table 1, participants with strong adherence to the DASH diet indicated a significant decrease in the mean of FBS. On the other hand, this table demonstrated that moderate adherence to the DASH diet had an inverse association with mean of BMI, waist circumference, waist-tohip ratio, body fat percentage, and visceral fat and had a positive association with physical activity, but none of them were statistically significant $(p>0.05)$.

The average intake of macronutrients, vitamins, and minerals in different quartiles of the adherence to the DASH diet scores indicated in Table 2. As shown in Table 2, the increase in adherence to the DASH diet had a significant relationship with a higher intake of carbohydrate, protein, EPA, DHA, vitamin D, vitamin $C$, and vitamin $A(p<0.05)$. This table also showed that individuals with higher adherence to the DASH diet had a significant decrease in the consumption of fat, Zinc, and vitamin B12. Besides, the strong following of this dietary pattern had a significant association with an increase in the intake of chromium, potassium, and iron $(p<0.05)$.

\section{Table 2}

Dietary intakes by quartile $(\mathrm{Q})$ categories of DASH dietary pattern scores in a sample of women with and without PCOS

\section{Adherence to the DASH diet and PCOS}

Odds ratio and $95 \%$ confidence interval for polycystic ovary syndrome between quartiles of adherence to the DASH diet (adjusted for potential confounders) demonstrated in Table 3.Three models are considered in this table with adjusting for potential confounders. Model 1 (that was just adjusted for Energy intake) demonstrated participants in the highest adherence to the DASH diet, had the lowest risk of developing PCOS, which was statistically significant $(\mathrm{OR}=0.17 ; 95 \% \mathrm{Cl} 0.07,0.41$; There was a significant decreasing trend in the odds of PCOS across increasing quintiles of the DASH diet ( $p$-trend $<0.001$ ).

This result remained significant after further adjustments for education, chronic disease history, socioeconomic status, diet history, marital status, pregnancy history, physical activity, and body fat percentage in addition to energy intake, in model 2 ( $\mathrm{OR}=0.18 ; 95 \% \mathrm{Cl} 0.07,0.47$; $\mathrm{p}$-trend $<0.001)$. Also, we found that adherence to the DASH diet could significantly decrease the odds of PCOS in the third model which was adjusted for visceral fat, besides those mentioned in model 2 , $(\mathrm{OR}=0.17 ; 95 \% \mathrm{Cl} 0.07$, 


\begin{tabular}{|c|c|c|c|c|c|}
\hline \multicolumn{6}{|c|}{ DASH diet } \\
\hline & Q1 & Q2 & Q3 & Q4 & P_Value \\
\hline & Mean \pm SE & Mean \pm SE & Mean \pm SE & Mean \pm SE & \\
\hline Fat (gr) & $78.04 \pm 24.90$ & $61.19 \pm 32.78$ & $60.04 \pm 28.36$ & $58.05 \pm 28.64$ & 0.062 \\
\hline $\begin{array}{l}\text { Carbohydrate } \\
\text { (gr) }\end{array}$ & $300.90 \pm 105.89$ & $302.66 \pm 136.8$ & $313.88 \pm 116.15$ & $329.56 \pm 106.58$ & $<0.001$ \\
\hline Protein (gr) & $85.16 \pm 26$ & $85.47 \pm 41.67$ & $79.71 \pm 28.42$ & $82.34 \pm 24.90$ & $<0.001$ \\
\hline Cholesterol & $115.11 \pm 216.40$ & $114.87 \pm 223.63$ & $114.31 \pm 249.29$ & $113.04 \pm 262.01$ & 0.232 \\
\hline EPA & $0.0096 \pm 0.014$ & $0.0172 \pm 0.029$ & $0.0172 \pm 0.016$ & $0.0299 \pm 0.027$ & $<0.001$ \\
\hline DHA & $0.025 \pm 0.037$ & $0.044 \pm 0.076$ & $0.044 \pm 0.043$ & $0.077 \pm 0.071$ & $<0.001$ \\
\hline Vitamin A & $15.25 \pm 11.63$ & $21.60 \pm 19.75$ & $20.46 \pm 20.42$ & $25.77 \pm 27.21$ & 0.016 \\
\hline Vitamin D & $1.41 \pm 1.7$ & $1.25 \pm 1.91$ & $1.19 \pm 1.03$ & $1.67 \pm 2.2$ & 0.008 \\
\hline Vitamin C & $164.2 \pm 95.3$ & $180.0 \pm 119.5$ & $213.9 \pm 150.4$ & $234.1 \pm 123.8$ & $<0.001$ \\
\hline Vitamin B12 & $4.23 \pm 2.25$ & $3.56 \pm 2.32$ & $3.48 \pm 1.95$ & $3.96 \pm 2.49$ & 0.005 \\
\hline lodine & $0.0002 \pm 0.0001$ & $0.0003 \pm 0.0002$ & $0.0003 \pm 0.0002$ & $0.0006 \pm 0.0004$ & 0.413 \\
\hline Zinc & $9.14 \pm 2.05$ & $9.12 \pm 4.44$ & $8.71 \pm 3.57$ & $8.63 \pm 3.21$ & 0.002 \\
\hline chrome & $0.44 \pm 0.01$ & $0.48 \pm 0.03$ & $0.49 \pm 0.04$ & $0.52 \pm 0.06$ & $<0.001$ \\
\hline $\mathrm{Fe}$ & $29.26 \pm 23.89$ & $47.83 \pm 95.66$ & $42.96 \pm 59.98$ & $52.38 \pm 37.71$ & 0.001 \\
\hline Potassium & $3133.0 \pm 1041.1$ & $\begin{array}{l}3424 . \\
2 \pm 1497.2\end{array}$ & $\begin{array}{l}37132 . \\
2 \pm 1820.5\end{array}$ & $4047.7 \pm 1633.8$ & $<0.001$ \\
\hline
\end{tabular}

$0.47 ; p$-trend $=0.000$ ). As it was indicated in all models, this decreasing trend was significant and $p$-trend $<0.05$.

\section{Table 3}

Multivariate adjusted odds ratio (OR) and 95\% confidence interval (CI) for PCOS among Quartile of DASH diet scores 


\section{DASH diet}

\begin{tabular}{|c|c|c|c|c|c|c|}
\hline & Q1 & Q2 & Q3 & Q4 & & $\mathrm{p}$-trend \\
\hline Model 11 & 1 & $1.02(0.42-2.49)$ & $0.24(0.11-0.54)$ & $0.17(0.07-0.41)$ & $<0.001$ & \\
\hline Model $2^{2}$ & 1 & $1.22(0.47-3.18)$ & $0.25(0.10-0.58)$ & $0.18(0.07-0.47)$ & $<0.001$ & \\
\hline Model $3^{3}$ & 1 & $1.21(0.46-3.16)$ & $0.24(0.10-0.58)$ & $0.18(0.07-0.47)$ & $<0.001$ & \\
\hline
\end{tabular}

${ }^{1}$ Adjusted for Energy intake

${ }^{2}$ Adjusted for Energy intake, Education, Chronic disease history, Socioeconomic, Diet history, Marital status, Pregnancy history, Physical activity, and Body fat percentage

${ }^{3}$ Adjusted for energy intake, Education, Chronic disease history, socioeconomic, Diet history, Marital status, Pregnancy history, Physical activity, Body fat percentage, and Visceral fat mass

\section{Discussion}

This study scrutinized the association between adherence to the DASH diet and the risk of PCOS. As we found, this study indicated that an increase in adherence to the DASH diet had a significant relation with 82 percent decreasing the chances of developing polycystic ovary syndrome. Also, this study indicated higher following of this dietary pattern had a significant association with an increase in intake of EPA [32], DHA [33], vitamin D [34, 35], vitamin C [36], vitamin A, chromium [37], potassium, and iron which their beneficial effects on PCOS have been noted in previous studies. Also, this study indicated that adherence to this dietary pattern may have a useful effect on the reduction of the level of FBS as a metabolic feature of PCOs.

A significant reverse association was found between adherence to the DASH diet and the chance of getting PCOS after full adjustments. Also, decreasing the FBS level had a significant relation with the strong following of the DASH diet. A cross-sectional study conducted by Alessa et al. indicated that adherence to the DASH diet as a healthful dietary pattern had a significant association with higher concentrations of sex-hormone-binding globulin (SHBG) [38] which may decrease the level of free testosterone and have a useful effect on PCOS.

Other studies in line with our findings were two randomized clinical trial study demonstrated that adherence to the DASH diet had beneficial effects on BMI, anti-Müllerian hormone (AMH), insulin metabolism, SHBG [39], losing weight, reduction in serum androstenedione, and increase in antioxidant status [23]. As it was found, some components of the DASH diet including vegetables, fruits, nuts and legumes, and low-fat dairy products, contain high amounts of antioxidants, dietary fiber, phytoestrogens, magnesium, and calcium, that their useful effects on PCOS which have been mentioned in previous studies $[22,40]$. On the other hand, calcium as an important micronutrient, which is found abundantly in low-fat dairy products, can increase insulin sensitivity [41]. Moreover, increased intake of calcium can be 
effective on weight loss which in turn would result in fecal fat excretion. weight loss can decrease insulin resistance [42] which may decrease the P450c17a enzyme activity and as a result decrease in ovarian androgen production occurs [43]. Insulin resistance and hyperinsulinemia as two pathogenesis factors in PCOS, by stimulation of androgen synthesis through Theca cells and reducing SHBG, may lead to hyperandrogenaemia $[44,45]$. Therefore, as a possible mechanism DASH diet which is rich in nuts that contain omega-3, has a protective effect on PCOS by increasing insulin sensitivity and SHBG level [4547].

There are several strengths in this study. First of all, to the best of our knowledge, this study is one of the first investigations in progress which to assess the relationship between adherence to DASH diet score and risk of PCOS in newly diagnosed patients in a Middle Eastern country.

Moreover, we considered newly diagnosed PCOS women as the case group to reduce the possibility of a conscious choice of a healthy diet by women with PCOS. Furthermore, a wide range of confounders, especially body composition in addition to energy intake was examined in this study that was not controlled in other similar articles. To reduce the self-reporting error, FFQ was filled out by a blinded trained dietitian to decide whether participants be in a case or a control group.

\section{Conclusion}

In conclusion, we found that adherence to a DASH diet had a reduction and protective effect on the chance of getting PCOS in Iranian women. Also, the high adherence to this dietary pattern had an association with increased intake of some vitamin, minerals, and macronutrients which have a great effect on increasing insulin sensitivity and reproductive chances.

\section{Limitations}

The current study has some limitations, such as the risk of recall bias that may occur while using the semi-quantitative FFQ. Also, given that the present study is a case-control study and there is no ability to respond to the causality, so prospective studies need to be done.

\section{Abbreviations}

PCOS: Polycystic Ovary Syndrome; DASH:Dietary Approaches to Stop Hypertension; AMH:anti-Müllerian hormone; SHBG:sex-hormone-binding globulin; WC:waist circumference; HC:hip circumference; FFQ:food frequency questionnaire; TLGS:Tehran Lipid and Glucose Study; Cl:confidence interval.

\section{Declarations}

\section{Ethics approval and consent to participate}


After explaining the process of our study, each participant provided signed written "informed consent". The Human Research Ethics Committees of Shahid Sadoughi University of Yazd Medical Sciences approved the study protocol (IR.SSU.SPH.REC.1396.168).

\section{Consent for publication}

Applicable

\section{Availability of data and materials}

The datasets supporting the conclusions of this study are available from the corresponding author upon reasonable request.

\section{Competing interests}

The authors have no conflicts of interest to declare.

\section{Funding}

None

\section{Authors' contributions}

A. Panjeshahin contributed to the conception and design of the research. Also, contributed to the acquisition and analysis of the data and drafted the manuscript.

M. Hosseinzadeh contributed to the design of the research and contributed to the interpretation of the data.

A. Ghadiri-Anari contributed to the design of the research

All authors critically revised the manuscript, agree to be fully accountable for ensuring the integrity and accuracy of the work, and read and approved the final manuscript.

\section{Acknowledgements}

The authors gratefully acknowledge Education Department of Shahid Sadoughi University of medical science of Yazd and those students who participated in this study. 


\section{References}

1. Azziz R, Carmina E, Dewailly D, Diamanti-Kandarakis E, Escobar-Morreale HF, Futterweit W, Janssen OE, Legro RS, Norman RJ, Taylor AE. The Androgen Excess and PCOS Society criteria for the polycystic ovary syndrome: the complete task force report. Fertility sterility. 2009;91:456-88.

2. Sirmans SM, Pate KA. Epidemiology, diagnosis, and management of polycystic ovary syndrome. Clinical epidemiology. 2014;6:1.

3. ESHRE TR, Group A-SPCW. Revised 2003 consensus on diagnostic criteria and long-term health risks related to polycystic ovary syndrome. Fertility sterility. 2004;81:19-25.

4. Teede HJ, Misso ML, Costello MF, Dokras A, Laven J, Moran L, Piltonen T, Norman RJ. Recommendations from the international evidence-based guideline for the assessment and management of polycystic ovary syndrome. Human reproduction. 2018;33:1602-18.

5. Jalilian A, Kiani F, Sayehmiri F, Sayehmiri K, Khodaee Z, Akbari M. Prevalence of polycystic ovary syndrome and its associated complications in Iranian women: A meta-analysis. Iran J Reprod Med. 2015;13:591-604.

6. Hardiman P, Pillay O, Atiomo W. Erratum: Polycystic ovary syndrome and endometrial carcinoma (Lancet (May 24, 2003) 361 (1810-1812)). Lancet. 2003;362:1082-.

7. Pastore LM, Patrie JT, Morris WL, Dalal P, Bray MJ. Depression symptoms and body dissatisfaction association among polycystic ovary syndrome women. J Psychosom Res. 2011;71:270-6.

8. Goodarzi MO, Dumesic DA, Chazenbalk G, Azziz R. Polycystic ovary syndrome: etiology, pathogenesis and diagnosis. Nat Rev Endocrinol. 2011;7:219-31.

9. Palomba S, Santagni S, Falbo A, La Sala GB. Complications and challenges associated with polycystic ovary syndrome: current perspectives. Int J Womens Health. 2015;7:745-63.

10. Wang ET, Calderon-Margalit R, Cedars MI, Daviglus ML, Merkin SS, Schreiner PJ, Sternfeld B, Wellons M, Schwartz SM, Lewis CE. Polycystic ovary syndrome and risk for long-term diabetes and dyslipidemia. Obstetrics gynecology. 2011;117:6.

11. Franz MJ, VanWormer JJ, Crain AL, Boucher JL, Histon T, Caplan W, Bowman JD, Pronk NP. Weightloss outcomes: a systematic review and meta-analysis of weight-loss clinical trials with a minimum 1-year follow-up. Journal of the American Dietetic association. 2007;107:1755-67.

12. Parker M, Warren A, Nair S, Barnard M. Adherence to treatment for polycystic ovarian syndrome: A systematic review. PloS one. 2020;15:e0228586.

13. Norman R, Homan G, Moran L, Noakes M. Lifestyle choices, diet, and insulin sensitizers in polycystic ovary syndrome. Endocrine. 2006;30:35-43.

14. Barber TM, Franks S. Genetics of polycystic ovary syndrome. In Polycystic ovary syndrome. Volume 40: Karger Publishers; 2013: pp. 28-39.

15. Panjeshahin A, Abarghuee AS, Anari AG, Mohammadi M, Hosseinzadeh M. The Association between Empirically Derived Dietary Patterns and Polycystic Ovary Syndrome: A Case-Control Study. Nutrition 2020:110987. 
16. Panjeshahin A, Khosravi M, Hosseinzadeh M. Polycystic Ovary Syndrome and Dietary Patterns in Iran: A Review Study. Journal of Nutrition Food Security. 2019;4:58-65.

17. Costello MF, Misso ML, Wong J, Hart R, Rombauts L, Melder A, Norman RJ, Teede HJ. The treatment of infertility in polycystic ovary syndrome: a brief update. Aust N Z J Obstet Gynaecol. 2012;52:4003.

18. Teede HJ, Misso ML, Deeks AA, Moran LJ, Stuckey BG, Wong JL, Norman RJ, Costello MF, Groups GD. Assessment and management of polycystic ovary syndrome: summary of an evidence-based guideline. Med J Aust. 2011;195:65-112.

19. Vollmer WM, Sacks FM, Ard J, Appel LJ, Bray GA, Simons-Morton DG, Conlin PR, Svetkey LP, Erlinger TP, Moore TJ. Effects of diet and sodium intake on blood pressure: subgroup analysis of the DASHsodium trial. Ann Intern Med. 2001;135:1019-28.

20. Azadbakht L, Fard NRP, Karimi M, Baghaei MH, Surkan PJ, Rahimi M, Esmaillzadeh A, Willett WC. Effects of the Dietary Approaches to Stop Hypertension (DASH) eating plan on cardiovascular risks among type 2 diabetic patients: a randomized crossover clinical trial. Diabetes Care. 2011;34:55-7.

21. Asemi Z, Samimi M, Tabassi Z, Shakeri H, Sabihi SS, Esmaillzadeh A. Effects of DASH diet on lipid profiles and biomarkers of oxidative stress in overweight and obese women with polycystic ovary syndrome: A randomized clinical trial. Nutrition. 2014;30:1287-93.

22. Asemi Z, Esmaillzadeh A. DASH diet, insulin resistance, and serum hs-CRP in polycystic ovary syndrome: a randomized controlled clinical trial. Horm Metab Res. 2015;47:232-8.

23. Azadi-Yazdi M, Karimi-Zarchi M, Salehi-Abargouei A, Fallahzadeh H, Nadjarzadeh A. Effects of Dietary Approach to Stop Hypertension diet on androgens, antioxidant status and body composition in overweight and obese women with polycystic ovary syndrome: A randomised controlled trial. Journal of Human Nutrition and Dietetics 2016.

24. Foroozanfard F, Rafiei H, Samimi M, Gilasi HR, Gorjizadeh R, Heidar Z, Asemi Z. The effects of dietary approaches to stop hypertension diet on weight loss, anti-Mullerian hormone and metabolic profiles in women with polycystic ovary syndrome: A randomized clinical trial. 2017, 87:51-58.

25. Azadi-Yazdi M, Karimi-Zarchi M, Salehi-Abargouei A, Fallahzadeh H, Nadjarzadeh A. Effects of Dietary Approach to Stop Hypertension diet on androgens, antioxidant status and body composition in overweight and obese women with polycystic ovary syndrome: a randomised controlled trial. J Hum Nutr Diet. 2017;30:275-83.

26. Azadi-Yazdi M, Karimi-Zarchi M, Salehi-Abargouei A, Fallahzadeh H, Nadjarzadeh A. Effects of Dietary Approach to Stop Hypertension diet on androgens, antioxidant status and body composition in overweight and obese women with polycystic ovary syndrome: a randomised controlled trial. Journal of human nutrition dietetics. 2017;30:275-83.

27. Azadbakht L, Surkan PJ, Esmaillzadeh A, Willett WC. The Dietary Approaches to Stop Hypertension eating plan affects C-reactive protein, coagulation abnormalities, and hepatic function tests among type 2 diabetic patients. J Nutr. 2011;141:1083-8. 
28. Katcher HI. The metabolic and reproductive effects of whole grains and high fiber foods in metabolic syndrome and polycystic ovary syndrome. Metabolic Syndrome Related Disorders. 2008;6:77-9.

29. Hinderliter AL, Babyak MA, Sherwood A, Blumenthal JA. The DASH diet and insulin sensitivity. Curr Hypertens Rep. 2011;13:67-73.

30. Excoffon L, Guillaume Y, Woronoff-Lemsi M, André C. Magnesium effect on testosterone-SHBG association studied by a novel molecular chromatography approach. J Pharm Biomed Anal. 2009;49:175-80.

31. Edelstein S, Roscoe H, Silverman D, Lynch M. Fostering Adolescent Obesity Prevention by Partnering College Student Mentors With a Diverse Middle/High School: A Pilot Study. Journal of Foodservice Business Research. 2015;18:171-81.

32. Salek M, Clark CC, Taghizadeh M, Jafarnejad S. N-3 fatty acids as preventive and therapeutic agents in attenuating PCOS complications. EXCLI J. 2019;18:558.

33. Kalgaonkar S, Almario R, Gurusinghe D, Garamendi E, Buchan W, Kim K, Karakas SE. Differential effects of walnuts vs almonds on improving metabolic and endocrine parameters in PCOS. Eur $\mathrm{J}$ Clin Nutr. 2011;65:386-93.

34. Thomson RL, Spedding S, Buckley JD. Vitamin D in the aetiology and management of polycystic ovary syndrome. Clin Endocrinol. 2012;77:343-50.

35. dehghani Firouzabadi R, Aflatoonian A, Modarresi S, Sekhavat L, MohammadTaheri S. Therapeutic effects of calcium \& vitamin D supplementation in women with PCOS. Complement Ther Clin Pract. 2012;18:85-8.

36. Hosseini S, Jahromi M, Sayadi M. Effect of vitamin C supplementation on the levels of related hormones in infertile women with polycystic ovary syndrome (PCOS) in Shiraz City. Int J Health Sci. 2014;2:61-70.

37. Amr N, Abdel-Rahim HE. The effect of chromium supplementation on polycystic ovary syndrome in adolescents. J Pediatr Adolesc Gynecol. 2015;28:114-8.

38. AlEssa HB, Malik VS, Yuan C, Willett WC, Huang T, Hu FB, Tobias DK. Dietary patterns and cardiometabolic and endocrine plasma biomarkers in US women. The American Journal of Clinical Nutrition. 2016;105:432-41.

39. Foroozanfard F, Rafiei H, Samimi M, Gilasi HR, Gorjizadeh R, Heidar Z, Asemi Z. The effects of dietary approaches to stop hypertension diet on weight loss, anti-Müllerian hormone and metabolic profiles in women with polycystic ovary syndrome: A randomized clinical trial. Clin Endocrinol. 2017;87:518.

40. Maktabi M, Jamilian M, Asemi Z. Magnesium-zinc-calcium-vitamin D co-supplementation improves hormonal profiles, biomarkers of inflammation and oxidative stress in women with polycystic ovary syndrome: a randomized, double-blind, placebo-controlled trial. Biol Trace Elem Res. 2018;182:21-8.

41. Onakpoya IJ, Perry R, Zhang J, Ernst E. Efficacy of calcium supplementation for management of overweight and obesity: systematic review of randomized clinical trials. Nutrition reviews. 2011;69:335-43. 
42. PASQUALI R, ANTENUCCI D, CASIMIRRI F, VENTUROLI S, PARADISI R, FABBRI R, BALESTRA V, MELCHIONDA N. BARBARA L: Clinical and hormonal characteristics of obese amenorrheic hyperandrogenic women before and after weight loss. The Journal of Clinical Endocrinology Metabolism. 1989;68:173-9.

43. Jakubowicz DJ, Nestler JE. 17a-Hydroxyprogesterone responses to leuprolide and serum androgens in obese women with and without polycystic ovary syndrome after dietary weight loss. The Journal of Clinical Endocrinology Metabolism. 1997;82:556-60.

44. Oner G, Muderris I. Efficacy of omega-3 in the treatment of polycystic ovary syndrome. J Obstet Gynaecol. 2013;33:289-91.

45. Ardawi MSM, Rouzi AA. Plasma adiponectin and insulin resistance in women with polycystic ovary syndrome. Fertil Steril. 2005;83:1708-16.

46. Sadeghi A, Djafarian K, Mohammadi H, Shab-Bidar S. Effect of omega-3 fatty acids supplementation on insulin resistance in women with polycystic ovary syndrome: Meta-analysis of randomized controlled trials. Diabetes Metab Syndr. 2017;11:157-62.

47. Kalgaonkar S, Almario R, Gurusinghe D, Garamendi E, Buchan W, Kim K, Karakas SE. Differential effects of walnuts vs almonds on improving metabolic and endocrine parameters in PCOS. Eur J Clin Nutr. 2011;65:386.

\section{Table}

Due to technical limitations, table 1 is only available as a download in the Supplemental Files section.

\section{Supplementary Files}

This is a list of supplementary files associated with this preprint. Click to download.

- table1.docx

- figure.S1.docx

- tableS1.docx 